\title{
On the Harmonic Problem with Nonlinear Boundary Integral Conditions
}

\author{
Saker Hacene \\ L.M.A. Department of Mathematics, Faculty of Sciences, University of Badji Mokhtar, P.O. Box 12, 23000 Annaba, Algeria
}

Correspondence should be addressed to Saker Hacene; h_saker@yahoo.fr

Received 18 November 2013; Revised 7 January 2014; Accepted 8 January 2014; Published 23 February 2014

Academic Editor: Shamsul Qamar

Copyright (C) 2014 Saker Hacene. This is an open access article distributed under the Creative Commons Attribution License, which permits unrestricted use, distribution, and reproduction in any medium, provided the original work is properly cited.

In the present work, we deal with the harmonic problems in a bounded domain of $\mathbb{R}^{2}$ with the nonlinear boundary integral conditions. After applying the Boundary integral method, a nonlinear boundary integral equation is obtained; the existence and uniqueness of the solution will be a consequence of applying theory of monotone operators.

\section{Introduction}

For the harmonic problem, the simplest boundary condition we can impose specifies $u$ at all points on the boundary $\Gamma$ and is known as the Dirichlet boundary condition. The Dirichlet problem for the Laplace equation can easily be solved using the boundary integral equation [1]. If the normal derivative of $u$, that is, $\partial u / \partial n$, where $n$ is the outward normal to the boundary $\Gamma$, is specified at all points on the boundary $\Gamma$, that is, the Neumann boundary condition, with $\int_{\Gamma}(\partial u / \partial n) d s=0$, then given the value of $u$ at one point on $\Gamma$ enables a unique solution to be obtained [1].

In this work, we impose more general boundary conditions, namely, the nonlinear integral equation of Urysohn type $[2,3]$.

Much attention has been paid to the resolution of boundary value problems for partial differential operators with nonlinear boundary conditions by the method of integral equations in many directions (see, e.g., Atkinson and Chandler $[4,5]$ and Ruotsalainen and Wendland [6]).

Problems involving nonlinearities form a basis of mathematical models of various steady-state phenomena and processes in mechanics, physics, and many other areas of science. Among these is the steady-state heat transfer. Also some electromagnetic problems contain nonlinearities in the boundary conditions, for instance, problems where the electrical conductivity of the boundary is variable [7]. Further applications arise in heat radiation and heat transfer $[7,8]$.
In the present paper, we look for the solution of the Laplacian equation with nonlinear data of the form

$$
\begin{gathered}
\Delta u(x)=0, \quad x \in \Omega, \\
\frac{\partial u}{\partial n}(x)+\int_{\Gamma} K(x, y, u(y)) d s_{y}=f(x), \quad x \in \Gamma .
\end{gathered}
$$

We recall that the nonlinear boundary integral operator defined by

$$
A(x, u(x))=\int_{\Gamma} K(x, y, u(y)) d s_{y}, \quad x \in \Gamma,
$$

is the nonlinear integral operator of Urysohn type.

In (1), we assume $\Omega$ is an open bounded region in $\mathbb{R}^{2}$ with a smooth boundary $\Gamma=\partial \Omega$, and

$$
f: \Gamma \longrightarrow \mathbb{R}, \quad K: \Gamma \times \Gamma \times \mathbb{R} \longrightarrow \mathbb{R}
$$

are given real value functions.

By the Green representation formula, we formulate a nonlinear integral equation on the boundary $\Gamma$ of the domain $\Omega$. Under some assumptions on the Kernel of the nonlinear integral equation of Urysohn $K(x, y, u)$ we prove the existence and uniqueness of the solution. 


\subsection{Definitions and Notations}

Definition 1 (see $[1,9]$ ). Let $m \in \mathbb{N}$; one denotes by $H^{m}(\Omega)$ the Sobolev space:

$$
H^{m}(\Omega)=\left\{u \in L^{2}(\Omega) ; D^{\alpha} u \in L^{2}(\Omega),|\alpha| \leq m\right\} .
$$

Definition 2 (see $[1,9])$. Let $s \in \mathbb{R}$; one denotes by $H^{s}\left(\mathbb{R}^{n}\right)$ the Sobolev space:

$$
H^{s}\left(\mathbb{R}^{n}\right)=\left\{u \in L^{2}\left(\mathbb{R}^{n}\right) ;\left(1+|\xi|^{2}\right)^{s / 2}|F[u]| \in L^{2}\left(\mathbb{R}^{n}\right)\right\} ;
$$

and the associated norm:

$$
\|u\|_{H^{s}}=\left(\int_{\mathbb{R}^{n}}\left(1+|\xi|^{2}\right)^{s}|F[u]|^{2} d \xi\right)^{1 / 2}
$$

with $F[\cdot]$ the Fourier transform.

Definition 3 (see $[1,9]$ ). Let $\Omega \subset \mathbb{R}^{n}$ a bounded domain and $\Gamma:=\partial \Omega$; one defined

$$
\begin{aligned}
& H^{s}(\Omega)=\left\{u \mid \Omega: u \in H^{s}\left(\mathbb{R}^{n}\right)\right\}, \quad s \in \mathbb{R} \\
& H^{s}(\Gamma)= \begin{cases}\left\{\left.u\right|_{\Gamma}: u \in H^{s+(1 / 2)}\left(\mathbb{R}^{n}\right)\right\}, & s>0 \\
L^{2}(\Gamma), & s=0 \\
\left(H^{-s}(\Gamma)\right)^{\prime} & \text { (dual space), } s<0 .\end{cases}
\end{aligned}
$$

Definition 4 (see $[1,9])$. The Fichera trace spaces $H^{s}(\Gamma)$, for $0<s<1$, is defined to be the completion of

$$
C_{s}^{0}(\Gamma):=\left\{\varphi \in C^{0}(\Gamma):\|\varphi\|_{H^{s}(\Gamma)}<\infty\right\},
$$

with respect to the norm

$$
\|u\|_{H^{s}(\Gamma)}:=\left\{\|u\|_{L^{2}(\Gamma)}^{2}+\iint_{\Gamma} \frac{|u(x)-u(y)|^{2}}{|x-y|^{1+2 s}} d s_{x} d s_{y}\right\}^{1 / 2} .
$$

\section{The Boundary Integral Method}

2.1. Representative Formula and Boundary Operator. We introduce the fundamental solution of the Laplacian operator in the plane defined by:

$$
E(x, y)=\frac{1}{2 \pi} \log |x-y| .
$$

We first consider some standard boundary integral operators.

For $x \in \Omega$, the single layer potential is

$$
S_{\Omega} u(x):=-\int_{\Gamma} E(x, y) u(y) d s_{y}
$$

and the double layer potential is

$$
D_{\Omega} u(x):=\int_{\Gamma} u(y) \frac{\partial}{\partial n_{y}} E(x, y) d s_{y} .
$$

Using Green's identity for harmonic functions, we get

$$
u(x)=\int_{\Gamma} u(y) \frac{\partial}{\partial n_{y}} E(x, y) d s_{y}-\int_{\Gamma} \frac{\partial u(y)}{\partial n_{y}} E(x, y) d s_{y}
$$

for $x \in \Omega$, which can be written as

$$
u(x)=D_{\Omega} u(x)+S_{\Omega} \frac{\partial u(x)}{\partial n}, \quad \text { for } x \in \Omega .
$$

Sending in (15) $x \rightarrow \Gamma$. The continuity of the simple layer potential $S_{\Omega}$ and the jump relation of the double layer potential $D_{\Omega}$. we can write the integral equation on the boundary as follows:

$$
u(x)-D u(x)=S \frac{\partial u(x)}{\partial n}, \quad x \in \Gamma
$$

where

$$
\begin{array}{ll}
S \frac{\partial u(x)}{\partial n}:=-2 \int_{\Gamma} E(x, y) \frac{\partial u(y)}{\partial n} d s_{y}, & x \in \Gamma, \\
D u(x):=2 \int_{\Gamma} u(y) \frac{\partial}{\partial n_{y}} E(x, y) d s_{y}, & x \in \Gamma .
\end{array}
$$

Clearly, if $u \in H^{1}(\Omega)$ is the solution of (1), then the Cauchy data $\left.u\right|_{\Gamma}$ and $\partial u /\left.\partial n\right|_{\Gamma}$ satisfies the integral equation (16).

Then the boundary conditions

$$
\frac{\partial u}{\partial n}(x)=-A(x, u(x))+f(x)
$$

yield

$$
u(x)-D u(x)=-S(A(x, u(x)))+S f(x), \quad x \in \Gamma .
$$

Equation (19) can be written as

$$
(I-D) u(x)+S(A(x, u(x)))=S f(x), \quad x \in \Gamma .
$$

Conversely, if $\left.u\right|_{\Gamma}$ solves (20), then the solution of (1) can be given by the representation formula (15) and will satisfy

$$
\frac{\partial u}{\partial n}(x)=-A(x, u(x))+f(x)
$$

due to (20). For studying the solvability of the nonlinear equation (20), we give some assumptions to be made here.

(H1) We assume a $\operatorname{diam}(\Omega)<1$.

(H2) The Kernel $K(\cdot, \cdot, \cdot)$ of the Urysohn operator is a Caratheodory function [3]

(H3) We assume that $\partial K(x, y, u) / \partial u$ is measurable satisfying

$$
0<a \leq \frac{\partial K(x, y, u)}{\partial u} \leq b<+\infty
$$

for some constants $a$ and $b$. 
Remark 5. (1) The operator $S$ may have eigenfunctions [1]; then $(\mathrm{H} 1)$ ensures that the integral operator

$$
S: H^{s}(\Gamma) \longrightarrow H^{s+1}(\Gamma)
$$

is an isomorphism for every $s \in \mathbb{R}$ and

$$
(S \mu, \mu) \geq c\|\mu\|_{H^{-1 / 2}}^{2}
$$

for all $\mu \in H^{-1 / 2}$ with some positive constant $c>0$ [1]. By $(\cdot, \cdot)$ we denote the $L^{2}(\Gamma)$ scalar product.

(2) The Kernel $K(\cdot, \cdot, \cdot)$ is a Caratheodory function $(\mathrm{H} 2)$; that is, $K(\cdot, \cdot, u)$ is measurable for all $u \in \mathbb{R}$ and $K(x, y, \cdot)$ is continuous for almost all $x, y \in \Gamma$.

(3) The assumption (H3) implies that the Nemytskii operator

$$
A: L^{2}(\Gamma) \longrightarrow L^{2}(\Gamma)
$$

is Lipschitz continuous and strongly monotonous such that

$$
\begin{array}{ll}
(A u-A v, u-v) \leq b & \operatorname{mes}(\Gamma)\|u-v\|_{0}^{2}, \\
(A u-A v, u-v) \geq a & \operatorname{mes}(\Gamma)\|u-v\|_{0}^{2},
\end{array}
$$

for all $u, v \in L^{2}(\Gamma)$.

Theorem 6. Let assumptions (H1), (H2), and (H3) hold. Then, for every $f \in H^{-1 / 2}$ the nonlinear boundary integral equation (20) has a unique solution in $H^{1 / 2}(\Gamma)$.

Proof. The proof follows from the well-known theorem by Browder and Minty on monotone operators $[6,10]$.

Since the simple layer potential operator on $\Gamma$

$$
S: H^{-1 / 2}(\Gamma) \longrightarrow H^{1 / 2}(\Gamma)
$$

is an isomorphism, it is sufficient to consider the unique solvability of the following equation:

$$
B u(x):=S^{-1}(I-D) u(x)+A(x, u(x))=f(x), \quad x \in \Gamma .
$$

We will prove that the operator

$$
B: H^{1 / 2}(\Gamma) \longrightarrow H^{-1 / 2}(\Gamma)
$$

is continuous and strongly monotonous.

(i) In the first we show that $B$ is continuous.

It is clear from the continuity of the mapping properties of the simple and double layer operators that

$$
S^{-1}(I-D): H^{1 / 2}(\Gamma) \longrightarrow H^{-1 / 2}(\Gamma)
$$

is continuous. And from (H3),

$$
A: H^{1 / 2}(\Gamma) \longrightarrow H^{-1 / 2}(\Gamma)
$$

is continuous. Hence the boundary integral operator

$$
B: H^{1 / 2}(\Gamma) \longrightarrow H^{-1 / 2}(\Gamma)
$$

is continuous. (ii) In the second we show that $B$ is strongly monotone operator.

The function $\mu \in H^{-1 / 2}(\Gamma)$ defined by

$$
\mu(x):=S^{-1}(I-D) u(x)
$$

for $u(x) \in H^{1 / 2}(\Gamma)$ is the normal derivative of the harmonic function

$$
w(x)=\int_{\Gamma} u(y) \frac{\partial}{\partial n_{y}} E(x, y) d s_{y}-\int_{\Gamma} \mu(y) E(x, y) d s_{y}
$$

for $x \in \Omega$; this means that $w$ satisfies the problem

$$
\begin{gathered}
\Delta w(x)=0, \quad x \in \Omega, \\
w(x)=u(x), \quad x \in \Gamma .
\end{gathered}
$$

Then Green's theorem yields

$$
\begin{aligned}
\left(S^{-1}(I-D) u, u\right) & =\int_{\Gamma} \mu u d s=\int_{\Gamma} \frac{\partial w}{\partial n} u d s \\
& =\int_{\Gamma} \frac{\partial w}{\partial n} w d s=\int_{\Omega}(\nabla w)^{2} d x
\end{aligned}
$$

Hence, for all $u, v \in H^{1 / 2}(\Gamma)$,

$$
\begin{aligned}
& \left(S^{-1}(I-D)(u-v), u-v\right) \\
& \quad=\int_{\Omega}\left(\nabla\left(w_{1}-w_{2}\right)\right)^{2} d x=\left|w_{1}-w_{2}\right|_{H^{1}(\Omega)}^{2},
\end{aligned}
$$

where $\left(w_{1}-w_{2}\right)$ denotes the harmonic function corresponding to the Cauchy data $u-v$ and $S^{-1}(I-D)(u-v)$.

On the other hand, we note that there exists $\left(\nu_{1}-v_{2}\right) \in$ $H^{-1 / 2}(\Gamma)$, such that

$$
S\left(v_{1}-v_{2}\right)=u-v
$$

on $\Gamma[1]$. Hence for all $x \in \Omega$, we have

$$
S_{\Omega}\left(v_{1}-v_{2}\right)=w_{1}-w_{2}
$$

The simple layer potential

$$
S_{\Omega}: H^{s}(\Gamma) \longrightarrow H^{s+(3 / 2)}(\Omega)
$$

is continuous, for all $s \in \mathbb{R}[1]$. Hence for $s=-3 / 2$, we find

$$
\begin{aligned}
\left\|w_{1}-w_{2}\right\|_{L^{2}(\Omega)} & \leq c_{1}\left\|v_{1}-v_{2}\right\|_{H^{-3 / 2}(\Gamma)} \\
& \leq c_{2}\|u-v\|_{H^{-1 / 2}(\Gamma)} \leq c_{3}\|u-v\|_{0},
\end{aligned}
$$

for some positive constants $c_{1}, c_{2}$, and $c_{3}$.

Hence we have

$$
\|u-v\|_{0} \geq \frac{1}{c_{3}}\left\|w_{1}-w_{2}\right\|_{L^{2}(\Omega)} .
$$


Then with (28) and (37) we get

$$
\begin{aligned}
(B u-B v, u-v)= & \left(S^{-1}(I-D)(u-v), u-v\right) \\
& +(A u-A v, u-v) \\
= & \left|w_{1}-w_{2}\right|_{H^{1}(\Omega)}^{2}+(A u-A v, u-v),
\end{aligned}
$$

and with (26) we get the inequality

$$
\begin{aligned}
(B u-B v, u-v) \geq & \left|w_{1}-w_{2}\right|_{H^{1}(\Omega)}^{2} \\
& +a \operatorname{mes}(\Gamma)\|u-v\|_{0}^{2} ;
\end{aligned}
$$

hence with (42) we have

$$
\begin{aligned}
(B u- & B v, u-v) \\
\geq & \left|w_{1}-w_{2}\right|_{H^{1}(\Omega)}^{2}+\frac{a \operatorname{mes}(\Gamma)}{c_{3}^{2}}\left\|w_{1}-w_{2}\right\|_{L^{2}(\Omega)}^{2} \\
\geq & \min \left\{1, \frac{a \operatorname{mes}(\Gamma)}{c_{3}^{2}}\right\} \\
& \times\left(\left|w_{1}-w_{2}\right|_{H^{1}(\Omega)}^{2}+\left\|w_{1}-w_{2}\right\|_{L^{2}(\Omega)}^{2}\right) \\
\geq & \min \left\{1, \frac{a \operatorname{mes}(\Gamma)}{c_{3}^{2}}\right\}\left\|w_{1}-w_{2}\right\|_{H^{1}(\Omega)}^{2} \\
\geq & c_{4}\|u-v\|_{H^{1 / 2}(\Gamma)}^{2}
\end{aligned}
$$

by the trace theorem $[1,9]$, which completes the proof.

Now we prove the regularity of the solution of the nonlinear boundary integral equation (20).

Theorem 7. For all $S f \in H^{s}(\Gamma), 1 / 2 \leq s \leq 3 / 2$, the unique solution of the nonlinear boundary integral equation (20) belongs to the space $H^{s}(\Gamma)$.

In the proof of this theorem we will need the following lemma.

Lemma 8. For every $u \in H^{s}(\Gamma), 0 \leq s \leq 1$, one has $A u \in$ $H^{s}(\Gamma)$ and the mapping $A: H^{s}(\Gamma) \rightarrow H^{s}(\Gamma)$ is bounded.

Proof. For $s=0, u \in H^{0}(\Gamma):=L^{2}(\Gamma)$ has already been proved.

For $s=1, u \in H^{1}(\Gamma), u$ is an absolutely continuous function. By assumption (H3) the function $A u(x)$ is Lipschitz continuous. Hence $A u(x)$ is also absolutely continuous function.

It remains to prove the case $0<s<1$; by the assumption (H3) and due to the definition of the Sobolev space in Definition 4, we have

$$
\iint_{\Gamma} \frac{|A u(x)-A u(y)|^{2}}{|x-y|^{1+2 s}} d s_{x} d s_{y} \leq b^{2}(\operatorname{mes}(\Gamma))^{2}\|u\|_{H^{s}(\Gamma)}^{2},
$$

which completes the proof of Lemma 8.
Proof of Theorem 7. Let $S f \in H^{s}(\Gamma), 1 / 2 \leq s \leq 3 / 2$, be given. By Theorem 6 , there exists a unique solution $u \in H^{1 / 2}(\Gamma)$ of the nonlinear boundary integral equation:

$$
(I-D) u+S A u=S f .
$$

Lemma 8 ensure that

$$
S f-S A u \in H^{S}(\Gamma)
$$

therefore

$$
(I-D) u \in H^{s}(\Gamma)
$$

This implies together with the Fredholm property of the double layer potential operator that $u \in H^{s}(\Gamma), 1 / 2 \leq s \leq 3 / 2$.

Example 9. Here we give an example to illustrate the theoretical results. We consider the harmonic problems:

$$
\begin{gathered}
\Delta u(x)=0, \quad x \in \Omega \\
\frac{\partial u}{\partial n}(x)+\int_{\Gamma}(2 u(y)+\sin u(y)) d s_{y}=f(x), \quad x \in \Gamma,
\end{gathered}
$$

where the nonlinear boundary integral equation of Urysohn type is defined by

$$
A u(x)=\int_{\Gamma}(2 u(y)+\sin u(y)) d s_{y}, \quad x \in \Gamma
$$

and the domain is

$$
\Omega=\left\{x=\left(x_{1}, x_{2}\right) x_{1}^{2}+x_{2}^{2}<r^{2}<\frac{1}{4}\right\} .
$$

Clearly, the nonlinearity satisfies our assumptions $\left(H_{1}\right),\left(H_{2}\right)$, and $\left(\mathrm{H}_{3}\right)$ such that

$$
\operatorname{diam}(\Omega)=2 r<1 .
$$

The Kernel $(2 u(y)+\sin u(y))$ of the nonlinear boundary integral equation of Urysohn type is a Caratheodory function. And

$$
\frac{\partial K(x, y, u)}{\partial u}=2+\cos u(y)
$$

is measurable satisfying

$$
1 \leq \frac{\partial(2 u(y)+\sin u(y))}{\partial u} \leq 3<+\infty
$$

implying that the Nemytskii operator

$$
A: L^{2}(\Gamma) \longrightarrow L^{2}(\Gamma)
$$

is Lipschitz continuous and strongly monotonous such that

$$
2 \pi r\|u-v\|_{0}^{2} \leq(A u-A v, u-v) \leq 6 \pi r\|u-v\|_{0}^{2}
$$

for all $u, v \in L^{2}(\Gamma)$. 


\section{Conflict of Interests}

The author declares that there is no conflict of interests regarding the publication of this paper.

\section{Acknowledgment}

The author would like to thank the referee for his very careful reading of the paper and his detailed comments and valuable suggestions which improved both the content and the presentation of this paper.

\section{References}

[1] G. C. Hsiao and W. Wendland, Boundary Integral Equations, Applied Mathematical Sciences, Springer, Berlin, Germany, 2008.

[2] A. jafarian, Z. Esmailzadeh, and L. Khoshbakhti, "A numerical method for solving nonlinear integral equation in the Urysohn form," Applied Mathematical Sciences, vol. 7, no. 28, pp. 13751385, 2013.

[3] M. Krasnosel'skii, Topological Methods in the Theory of Nonlinear Integral Equations, Macmillan, New York, NY, USA, 1964.

[4] K. Atkinson and G. Chandler, "Boundary integral equation methods for solving Laplace's equation with nonlinear boundary conditions," Mathematics of Computation, vol. 55, no. 192, pp. 451-472, 1990.

[5] K. Atkinson, The Numerical Solution of Integral Equations of the Second Kind, Cambridge University Press, Cambridge, UK, 1997.

[6] K. Ruotsalainen and W. Wendland, "On the boundary element method for some nonlinear boundary value problems," Numerische Mathematik, vol. 53, no. 3, pp. 299-314, 1988.

[7] R. Bialecki and A. J. Nowak, "Boundary value problems in heat conduction with nonlinear material and nonlinear boundary conditions," Applied Mathematical Modelling, vol. 5, no. 6, pp. 417-421, 1981.

[8] C. A. Brebbia, J. C. F. Telles, and L. C. Wrobel, Boundary Element Techniques, Springer, Berlin, Germany, 1984.

[9] R. A. Adams, Sobolev Spaces, Academic Press, New York, NY, USA, 1975.

[10] D. R. Smart, Fixed Point Theorems, Cambridge University Press, Cambridge, UK, 1980. 


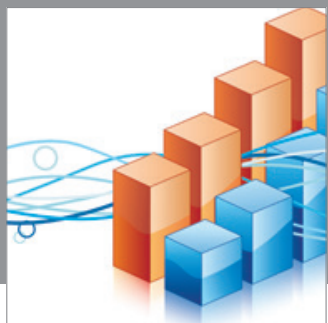

Advances in

Operations Research

mansans

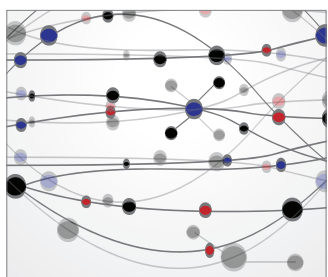

The Scientific World Journal
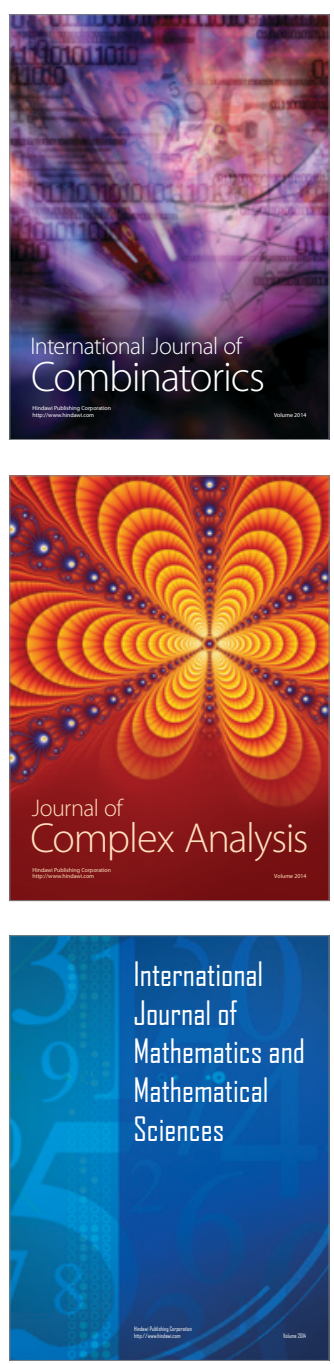
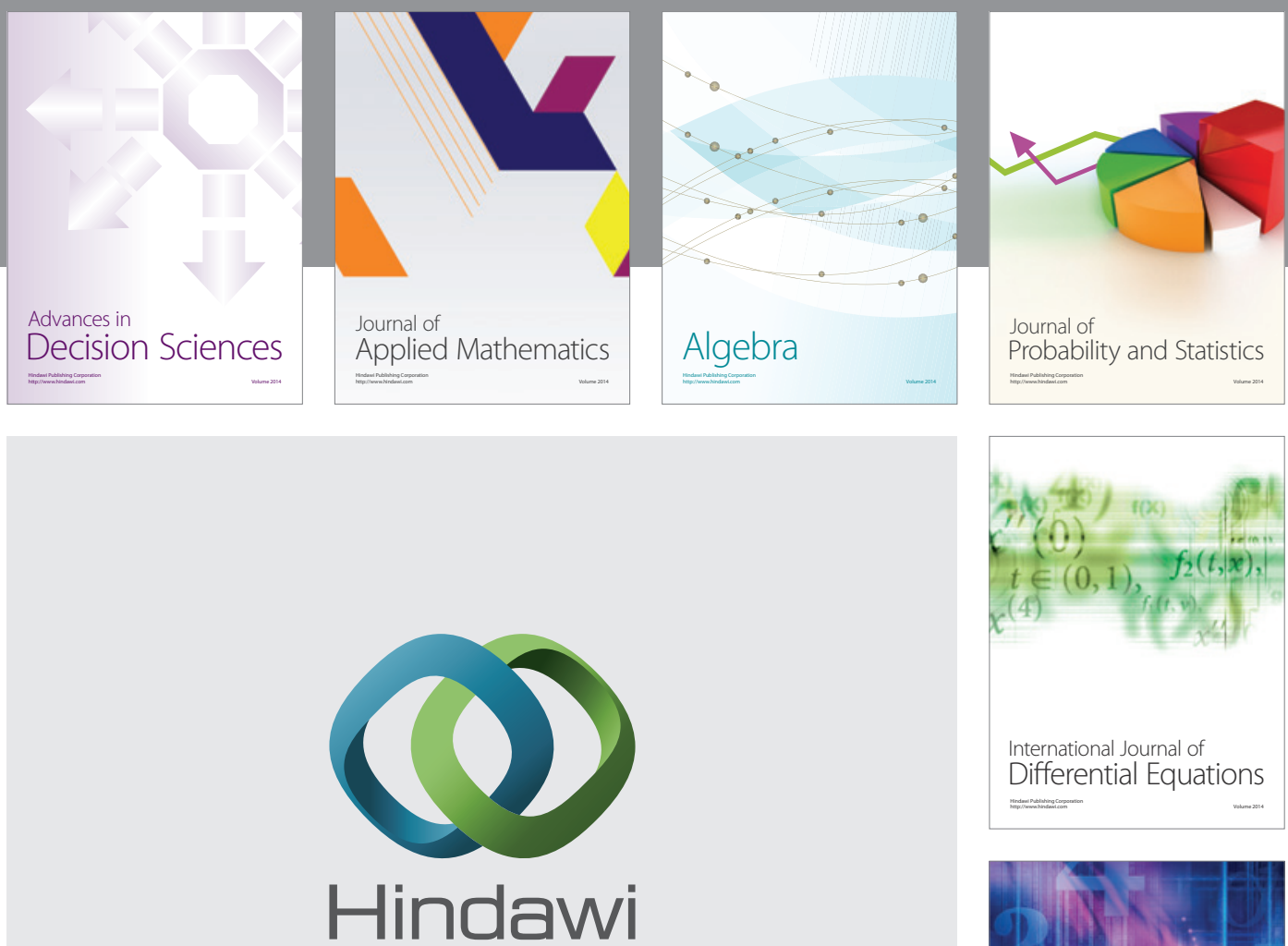

Submit your manuscripts at http://www.hindawi.com
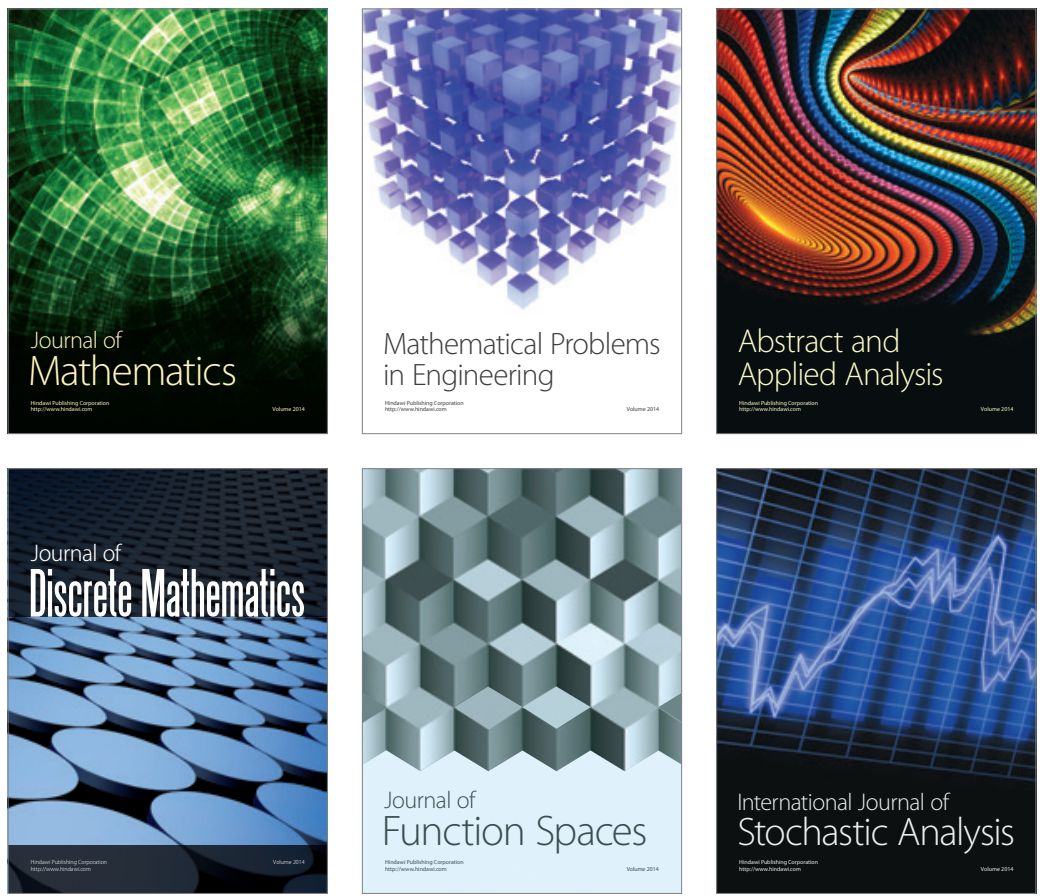

Journal of

Function Spaces

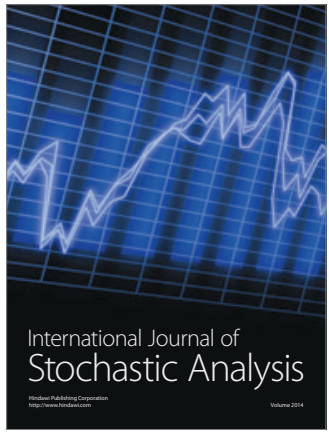

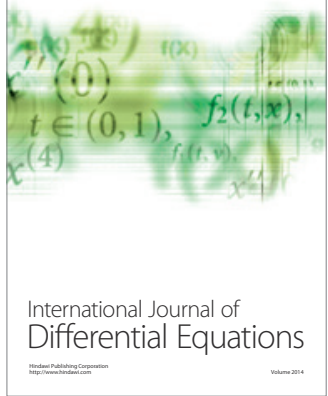
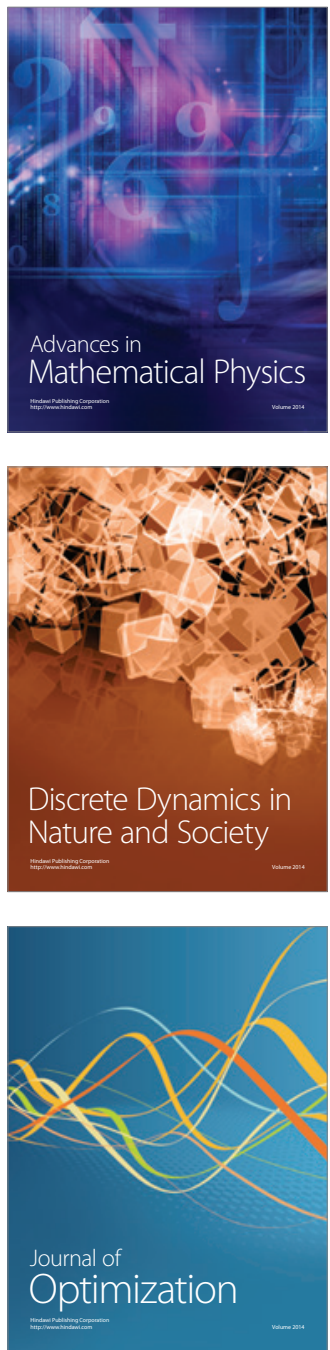